\title{
Segregación socioeconómica escolar en Chile: elección de la escuela por los padres y un análisis contrafactual teórico
}

\author{
Humberto Santos y Gregory Elacqua
}

RESUMEN

\begin{abstract}
En este artículo se examina la hipótesis de que la política de elección escolar ha incrementado la segregación socioeconómica en Chile. Se utiliza una base de datos georreferenciada de estudiantes y escuelas del Gran Santiago, para comparar la segregación real con la que se produciría en un escenario hipotético en que los estudiantes asisten a la escuela más cercana a su lugar de residencia. Los resultados indican que la segregación escolar es mayor en el escenario real que en el contrafactual, lo que sugiere que la interacción entre las preferencias de las familias y las barreras de entrada establecidas por las escuelas (cobros obligatorios y procesos de admisión selectivos) tienden a incrementar la segregación escolar más allá del efecto de la segregación residencial subyacente en la ciudad.
\end{abstract}




\section{I}

\section{Introducción}

En 1981, el sistema escolar chileno fue sometido a una reforma estructural decretada por el régimen militar (1973-1989). En primer lugar, el gobierno descentralizó la administración de las escuelas públicas, transfiriéndola del gobierno central a los municipios. En segundo lugar, cambió el método de financiamiento de las escuelas públicas y privadas. Las escuelas públicas continuaron recibiendo fondos del gobierno central, pero se comenzó a pagar a los municipios un subsidio (voucher) por cada estudiante matriculado en sus establecimientos. Las escuelas privadas (con y sin fines de lucro) que no cobraban mensualidades comenzaron a recibir el mismo subsidio por estudiante que las escuelas públicas. Estas políticas se introdujeron como una forma de aumentar la autonomía de las escuelas públicas y promover la eficiencia, al estimular la competencia por atraer a una mayor cantidad de estudiantes.

Los principales componentes del sistema universal de elección escolar no han cambiado en más de tres décadas. Las únicas modificaciones relevantes de las normas que regulan el programa de vouchers se introdujeron en 1994, cuando el gobierno promulgó una ley que permitía a todas las escuelas privadas subvencionadas y a las escuelas secundarias públicas el cobro de mensualidades, y en 2008, cuando el gobierno prohibió a las escuelas primarias el uso de entrevistas a los padres y pruebas durante los procesos de admisión. En ese mismo año, el gobierno introdujo también un voucher adicional (un $50 \%$ por sobre el valor base del subsidio), dirigido a aquellos estudiantes clasificados como desfavorecidos por el Ministerio de Educación (MINEDUC). El objetivo de esas modificaciones fue mejorar el diseño del programa de vouchers, ofreciendo a las escuelas incentivos para atraer a un alumnado diverso y ampliando el acceso de los padres a un conjunto variado de opciones de instrucción e información objetiva sobre las escuelas.

Si bien existen numerosos trabajos en la literatura en los que se han estudiado los efectos del programa chileno de vouchers en el rendimiento escolar (véanse, por ejemplo, Auguste y Valenzuela, 2004; Gallego, 2002; Hsieh y Urquiola, 2006), los efectos de la elección escolar en la segregación socioeconómica se han abordado solo recientemente (por ejemplo, Bellei, De los Ríos y Valenzuela, 2014; Elacqua, 2012). Este tema constituye motivo de preocupación por dos razones principales. En primer lugar, existe evidencia que sugiere que el sistema escolar chileno presenta uno de los niveles más altos de segregación socioeconómica entre los países que participan en el Programa Internacional de Evaluación de Estudiantes (PISA) (OCDE, 2011). En segundo lugar, en un número considerable de estudios internacionales se muestra que la segregación escolar tiene efectos adversos en diferentes resultados, entre ellos el rendimiento académico (Hanushek, Kain y Rivkin, 2009; Hoxby, 2002; Kahlenberg, 2001; Zimmer y Toma, 2000), el embarazo adolescente, la probabilidad de deserción escolar (Guryan, 2004), el ambiente educativo en la escuela (Hanushek, Kain y Rivkin, 2004), resultados no cognitivos como el compromiso intelectual y la motivación (Crain y Strauss, 1985; Rothstein, 2004; Wells y otros, 2008) y los índices de compromiso cívico (Levinson y Levinson, 2003). Si bien existe un relativo consenso acerca de los altos niveles de segregación escolar y sus efectos negativos, hay menos acuerdo sobre cuánto contribuye a este resultado el programa nacional de vouchers. Por una parte, los críticos del sistema argumentan que la interacción entre las preferencias de las familias y las barreras de entrada establecidas por las escuelas tiende a incrementar la segregación escolar (Bellei, De los Ríos y Valenzuela, 2014). Por otra, quienes lo defienden afirman que la segregación escolar se explica sobre todo por la desigualdad en la distribución del ingreso en Chile y los altos niveles de segregación residencial que caracterizan a sus ciudades y barrios (Beyer y Eyzaguirre, 2014).

Para contribuir a este debate, se construyó una base de datos única, utilizando información georreferenciada de estudiantes y escuelas del Gran Santiago, con el objeto de estudiar el efecto de la elección escolar en la segregación socioeconómica. Específicamente, en este artículo se compara la segregación escolar real con el nivel de segregación que existiría en el caso hipotético de que los estudiantes asistieran a la escuela más cercana a su lugar de residencia. Si la elección de la escuela y otros componentes estructurales del sistema (en lugar de la segregación residencial) constituyen la fuerza impulsora de la segregación escolar, esta debería ser mayor en la situación real que en el escenario hipotético (no elección). Una de las características más interesantes del caso chileno es la coexistencia de diferentes tipos de proveedores de 
educación, pues permite a los investigadores analizar las disimilitudes en el funcionamiento de las escuelas públicas y las escuelas privadas subvencionadas con fines de lucro y sin fines de lucro. La evidencia sobre este tema es limitada, porque la mayoría de los sistemas escolares no proporcionan financiamiento público a las escuelas con fines de lucro. En la mayoría de los países, las escuelas continúan siendo financiadas y administradas por el gobierno y se requiere que las escuelas privadas no tengan fines de lucro (Elacqua, 2012; James, 1993).
El artículo se divide en seis secciones, incluida esta Introducción. En la siguiente sección se presenta una revisión de la literatura sobre los efectos de la elección escolar en la segregación en Chile. En la tercera se describe la metodología del estudio y en la cuarta se presentan los datos utilizados. En la quinta sección se detallan los principales resultados. Finalmente, en la sexta y última sección se presentan las conclusiones y se examinan las implicancias del estudio para la formulación de políticas públicas.

\section{II}

\section{Revisión de la literatura}

Los defensores del sistema de vouchers argumentan que, dados los altos niveles de segregación residencial en la mayoría de las ciudades, la posibilidad de elegir libremente la escuela puede reducir la segregación escolar al dar a los padres de bajos ingresos acceso a escuelas más integradas fuera de sus barrios (Moe, 2001). Algunos autores también afirman que, al disponer de información más objetiva sobre la calidad de las escuelas (por ejemplo, resultados en pruebas estandarizadas) que acerca de la calidad de los barrios, la elección de escuela puede depender menos del perfil sociodemográfico de las familias que la elección del barrio de residencia (Krysan, 2002). En consecuencia, los partidarios del sistema sostienen que las políticas de vouchers son más efectivas que los programas tradicionales de asignación geográfica para reducir la segregación socioeconómica ${ }^{1}$.

Por otra parte, los críticos de la elección escolar citan factores relacionados con la demanda y con la oferta de servicios educativos, que pueden influir en la concentración de estudiantes de niveles socioeconómicos similares dentro de las escuelas. Por la parte de la demanda, los escépticos sostienen que la composición social de la escuela es un atributo valorado por las familias. Por ejemplo, Elacqua, Buckley y Schneider (2006) encontraron que las familias de Santiago son más propensas a elegir la escuela sobre la base de su composición socioeconómica que de acuerdo con indicadores objetivos de rendimiento académico. Sin

\footnotetext{
${ }^{1}$ Véase un debate sobre los factores que influyen en la elección de la escuela y del barrio en Bifulco, Ladd y Ross (2009).
}

embargo, si la evaluación por parte de la familia de los atributos escolares varía conforme con sus propias características, los estudiantes de niveles socioeconómicos diferentes decidirán no asistir al mismo tipo de escuela. Por ejemplo, Gallego y Hernando (2009) mostraron que, entre las familias de mayores ingresos, la valoración de la distancia al elegir una escuela es menor, mientras que la de la calidad académica (medida por los resultados en las pruebas) es mayor con respecto al resto de las familias. Por último, una preocupación que surge es que los padres de niveles socioeconómicos más bajos no tengan los recursos necesarios para elegir la escuela de sus hijos: información sobre alternativas disponibles, redes, tiempo, capacidad para procesar la información, entre otros. Elacqua y Fábrega (2004), por ejemplo, hallaron que los padres con un bajo nivel de educación tienden a formar sus opiniones basados sobre todo en información que reciben de sus redes sociales (familia, vecinos, amigos, miembros de la iglesia, colegas y asociaciones de vecinos, entre otros) en comparación con los padres de un nivel de educación más alto. Sin embargo, la calidad de sus redes — medida según el nivel de instrucción de sus miembros- es baja en comparación con la de los padres de mayores ingresos.

Desde el punto de vista de la oferta, los críticos del sistema de vouchers argumentan que la competencia incentiva a algunas escuelas a seleccionar a los estudiantes más favorecidos, cuya educación es menos costosa de proveer. Por ejemplo, Hsieh y Urquiola (2006) encontraron que el sistema de vouchers introducido en Chile en 1981 generó un incremento de la estratificación escolar, especialmente debido al éxodo de los estudiantes de 
clase media desde las escuelas públicas a las escuelas privadas subvencionadas ${ }^{2}$. Uno de los principales efectos de la reforma de 1981 fue la diversificación de la oferta de escuelas privadas, debido principalmente al surgimiento de proveedores con fines de lucro, sector que en la actualidad representa un tercio de la matrícula escolar (Elacqua, Martínez y Santos, 2015).

La enseñanza con fines de lucro es objeto de un encendido debate. El principal argumento en favor de estas escuelas es que la búsqueda de la rentabilidad económica requiere que ellas se concentren en el cliente y esto supondría un mejor equilibrio entre las preferencias de los padres y la misión de la escuela. Si bien la diversidad de alternativas es fundamental para un sistema de elección escolar, los críticos afirman que esta diferenciación reduce la integración de los estudiantes con distintos orígenes sociales y culturales (Bellei, 2010). Quienes se oponen al sistema argumentan que, debido a que las escuelas con fines de lucro están orientadas a la rentabilidad, tratarán de reducir los costos dirigiéndose a los estudiantes más favorecidos y menos caros de educar (Levin, 2002). Algunos expertos sostienen también que las escuelas sin fines de lucro, que en Chile representan aproximadamente el 15\% de la matrícula total, están mejor preparadas para atender a los estudiantes desfavorecidos que las escuelas con fines de lucro, porque con frecuencia cuentan con donaciones y enfrentan costos laborales más bajos (como por ejemplo, voluntariado) para financiar las altas inversiones involucradas en la educación de estudiantes de bajos ingresos (Rose-Ackerman, 1996). Solo recientemente se han comenzado a analizar las diferencias de comportamiento entre los diversos tipos de proveedores privados en el sistema educativo chileno. Por ejemplo, Elacqua (2012) muestra que, al contrario de lo esperado, las escuelas con fines de lucro matriculan a un mayor porcentaje de estudiantes de bajos ingresos que las escuelas sin fines de lucro. Sin embargo, la segregación escolar es mayor dentro del sector con fines de lucro, lo que sugiere que estas escuelas buscan nichos de mercado en diferentes grupos socioeconómicos.

Existen dos mecanismos que las escuelas chilenas pueden utilizar para definir el tipo de estudiante que atenderán: el cobro obligatorio de mensualidades y

\footnotetext{
2 La Subvención Escolar Preferencial (SEP), establecida en 2008, cambió los incentivos para las escuelas: se introdujo un subsidio adicional para los estudiantes vulnerables y se prohibió a las escuelas el cobro de mensualidades a dichos estudiantes. La Ley General de Educación (LGE), también promulgada en 2008, prohibió las prácticas de selección en la enseñanza primaria (primero a sexto grado).
}

los procesos de admisión de estudiantes. El principal argumento en contra del cobro de mensualidades es que el pago obligatorio segmentaría el sector privado subvencionado sobre la base de la capacidad de pago de las familias. Bellei, De los Ríos y Valenzuela (2014) encontraron que, en promedio, cuanto mayor es el número de escuelas privadas que exigen el pago en un barrio, mayor es el nivel de segregación socioeconómica en las escuelas de ese barrio. De acuerdo con Elacqua (2012), las escuelas que cobran mensualidades tienen una menor proporción de estudiantes de bajos ingresos que las escuelas privadas subvencionadas gratuitas y las públicas. En este estudio también se constata que el sector privado subvencionado que cobra mensualidades está más segregado internamente que los sectores público y privado subvencionado gratuito. Sin embargo, algunos investigadores cuestionan estos resultados. Por ejemplo, Gallego y Hernando (2008) argumentan que la segregación se explica en mayor medida por factores vinculados a las preferencias de las familias y que la eliminación de los cobros solo tendría efectos moderados en la segregación escolar.

Con respecto al proceso de admisión escolar en Chile, la evidencia sugiere que, a pesar de que la Ley General de Educación (LGE) prohíbe la selección en la enseñanza primaria, muchas escuelas todavía realizan entrevistas a los padres y exigen que estos presenten documentos que acrediten su nivel de ingreso y su afinidad con determinadas creencias religiosas (por ejemplo, certificados de bautismo o de matrimonio religioso), sobre todo en el sector privado subvencionado. Muchas escuelas también requieren que los estudiantes rindan exámenes de ingreso (Bustos, Contreras y Sepúlveda, 2010; Carrasco y otros, 2014; Elacqua, Martínez y Santos, 2011).

Además de los factores directamente relacionados con la oferta y la demanda, en la mayoría de los sistemas escolares -independientemente del grado de libertad de elección que tienen las familias - la segregación residencial de las ciudades está vinculada con la segregación escolar. En el caso de Chile, existe evidencia de que un porcentaje considerable de padres tienden a elegir escuelas situadas cerca del lugar de residencia —en particular, en la enseñanza preescolar y primaria-, de manera que se establece una relación directa entre la composición social del barrio y la de la escuela. Por ejemplo, según Alves y otros (2012), el 70\% de los estudiantes de cuarto grado matriculados en las escuelas públicas y privadas subvencionadas del Gran Santiago viaja menos de 1,5 km para llegar al establecimiento escolar. Así, algunos autores han concluido que la segregación residencial 
es la principal causa que explica la segregación escolar (Beyer y Eyzaguirre, 2014; El Mercurio, 2013).

En este artículo se examina la hipótesis de que la política de elección escolar ha incrementado la segregación

\section{III}

\section{Metodología}

En este artículo, la estrategia empírica se basa en dos componentes principales. En primer lugar, se define el concepto de segregación y se analizan los diversos índices utilizados para medirla, que se basan en la literatura desarrollada para estudiar la segregación residencial y escolar entre minorías raciales (Duncan y Duncan, 1955; James y Taeuber, 1985; Massey y Denton, 1988; White, 1986; Zoloth, 1976). En segundo lugar, se presenta el diseño del escenario contrafactual y los supuestos en los que está basado.

\section{1. Índices de segregación}

En términos muy generales, la segregación es el grado en que dos o más grupos están aislados uno del otro dentro de un determinado espacio geográfico. De acuerdo con Massey y Denton (1988), existen cinco dimensiones por las cuales diferentes grupos pueden estar segregados: similitud, exposición, concentración, centralización y agrupación. De estas dimensiones, la similitud y la exposición han sido las más desarrolladas en la literatura y existen diversos índices alternativos para medirlas.

En primer lugar, se utilizaron las versiones de dos grupos (Duncan y Duncan, 1955) y múltiples grupos (Reardon y Firebaugh, 2002) del índice de disimilitud o índice de Duncan $(D)$. Al suponer la existencia de dos grupos ${ }^{3}$, uno definido como grupo minoritario (por ejemplo, estudiantes desfavorecidos desde el punto de vista económico o que pertenecen a un determinado grupo étnico) y el otro como grupo mayoritario (resto de los estudiantes), y definir $T$ y $P$ como el tamaño de la población total y la proporción del grupo minoritario en la ciudad, respectivamente, y $t_{i}$ y $p_{i}$ como los

3 Véase la expresión matemática para el índice de disimilitud de múltiples grupos en Reardon y Firebaugh (2002). La interpretación de este índice es similar a la de la versión de dos grupos. La diferencia es que en este caso se considera la distribución de n grupos, en vez de dividir a la población en dos categorías mutuamente excluyentes. socioeconómica en Chile, comparando la segregación real con la que existiría en el caso hipotético de que los estudiantes asistieran a la escuela más cercana a su lugar de residencia. mismos valores para la escuela $i$, este índice se define matemáticamente como:

$$
D=\sum t_{i}\left|p_{i}-P\right| / 2 T P(1-P)
$$

donde $D$ es la desviación media ponderada de la proporción de estudiantes del grupo minoritario en cada escuela de $P$, expresada como una fracción de su máximo. En consecuencia, el valor de $D$ varía entre 0 (no hay segregación) y 1 (segregación total). $D$ puede interpretarse como la proporción de todos los estudiantes, en cualquiera de los grupos, que deberían transferirse para que $p_{i}$ sea igual a $P$ para toda $i$, o en otras palabras, para que todas las escuelas de la ciudad tengan la misma composición social (Duncan y Duncan, 1955).

En segundo lugar, se utilizó el índice de exposición $(X)$, que se define como:

$$
E=\sum t_{i}\left(1-p_{i}\right) / T
$$

donde $T$ es el número total de estudiantes minoritarios en la ciudad y $t_{i}$ y $p_{i}$ son el número y la proporción de estudiantes pertenecientes a grupos minoritarios en la escuela $i$. El índice puede interpretarse como el porcentaje de estudiantes del grupo mayoritario que existe en la escuela típica a la que asiste un estudiante minoritario. A diferencia del índice de Duncan, este índice depende del tamaño relativo de los grupos. Esto significa que es sensible a la definición del grupo minoritario y, por lo tanto, no es simétrico (el índice no necesariamente tiene el mismo valor para los grupos minoritario y mayoritario).

Se emplea el índice de Duncan porque cumple con los criterios fundamentales de un índice de segregación (James y Taeuber, 1985), se utiliza ampliamente en la literatura empírica y tiene una interpretación directa que facilita la comparación con otros estudios nacionales e internacionales. Se emplea el índice de exposición porque su interpretación es más sencilla, lo que facilita 
el análisis de los resultados. Aunque existen otros índices de segregación empleados en la literatura que cumplen con propiedades más exigentes, en general no son fáciles de interpretar y se utilizan principalmente para realizar comparaciones a lo largo del tiempo y entre ciudades (Reardon y Firebaugh, 2002).

\section{El diseño del escenario contrafactual}

Existen varios estudios en los que se compara el nivel de segregación escolar observado con el nivel simulado bajo diferentes escenarios contrafactuales, en los que todos los estudiantes asisten a la escuela pública asignada por criterios geográficos (Bifulco, Ladd y Ross, 2009, para el distrito escolar de Durham, Carolina del Norte; Sohoni y Saporito, 2009, para los 22 distritos escolares más grandes de los Estados Unidos de América; Riedel y otros, 2010, para la ciudad de Wuppertal, Alemania) o a la escuela más cercana a su lugar de residencia (Allen, 2007, para Inglaterra; Östh, Andersson y Malmberg, 2013, para Suecia), sobre la base de información georreferenciada de las escuelas y las familias. Aunque se analizan sistemas con distintos grados de elección escolar, en los cinco estudios se encontró que el nivel de segregación escolar por raza, etnia, nivel socioeconómico y/o habilidad académica es mayor en la situación real —en la que las familias pueden elegir una escuela distinta de la asignada o de la más cercana a su lugar de residencia- que en el escenario contrafactual. En consecuencia, los autores concluyen que la posibilidad de elección exacerba la segregación escolar.

En este trabajo se emplea un método similar al utilizado por Allen (2007), quien simula un escenario contrafactual en que los estudiantes asisten a la escuela más cercana a su lugar de residencia. El nivel de segregación escolar en este escenario se explica completamente por la distribución geográfica de los estudiantes de diferente nivel socioeconómico dentro de la ciudad (segregación residencial) y por la localización de las escuelas. La diferencia entre este nivel de segregación y el nivel real es lo que Allen (2007) denomina segregación post-residencial, pues mide el efecto adicional de las elecciones de las familias que envían a sus hijos a una escuela distinta de la más cercana a su lugar de residencia, ya sea por decisión propia o porque esa escuela no está disponible para el estudiante.

Se reconoce que este escenario contrafactual puede no reflejar exactamente lo que ocurriría en ausencia de una política de elección escolar. Primero, se asume que las familias no pueden cambiar su lugar de residencia. Sin embargo, existe evidencia de que la elección del lugar donde vivir depende de la oferta educativa disponible ${ }^{4}$. De esa manera, en un caso hipotético en que los estudiantes deben asistir a la escuela más cercana, se esperaría - por lo menos a largo plazouna mayor movilidad residencial entre los padres que procuran acceder a las escuelas que se ajustan más a sus preferencias. Debido al costo que supone un cambio de residencia, este mecanismo de elección estaría disponible sobre todo para las familias de altos ingresos, lo que aumentaría los niveles de segregación residencial y escolar. Segundo, se asume que las escuelas no utilizan ningún mecanismo de selección y, en consecuencia, la distribución de los estudiantes se basa exclusivamente en el lugar de residencia. Tercero, se asume que no hay escuelas nuevas y que las escuelas existentes se mantienen dentro del sistema. Por último, los análisis contrafactuales no tienen en cuenta las restricciones de capacidad, dada la infraestructura actual de las escuelas.

\footnotetext{
${ }^{4}$ Por ejemplo, varias investigaciones muestran que el desempeño de las escuelas públicas tiene efectos significativos en el precio de las viviendas en los sistemas escolares que asignan a los estudiantes a las escuelas según su lugar de residencia (Black, 1999; Bayer, Ferreira y McMillan, 2007; Reback, 2005, para los Estados Unidos de América; Gibbons y Machin, 2003 y 2006, para el Reino Unido de Gran Bretaña e Irlanda del Norte; y Fack y Grenet, 2010, para Francia).
} 


\section{Datos utilizados}

Para el análisis empírico se utilizan datos sobre los estudiantes de cuarto grado en el Gran Santiago, relativos al año 2010. Los datos provienen de cuatro fuentes. En primer lugar, la encuesta para padres del Sistema de Medición de la Calidad de la Educación (SIMCE) contiene información sobre el nivel socioeconómico del estudiante, incluido el nivel educacional más alto alcanzado por sus padres ${ }^{5}$. En segundo lugar, el Sistema de Información General de Estudiantes (SIGE) del Ministerio de Educación (MINEDUC) incluye la dirección de la mayoría de los estudiantes de las escuelas públicas y privadas subvencionadas ${ }^{6}$. En tercer lugar, la información administrativa del MINEDUC contiene datos sobre las características de las escuelas, incluido el tipo de propiedad (con fines de lucro, sin fines de lucro) y el pago de mensualidades. Por último, el sitio web del MINEDUC contiene una base de datos con la dirección de todas las instituciones de educación preescolar, primaria y secundaria de Chile ${ }^{7}$.

La población objetivo consiste en los estudiantes que asisten a las escuelas públicas y privadas subvencionadas del Gran Santiago (69.014). De este universo, fue posible georreferenciar las direcciones de 31.645 estudiantes $(46 \%)^{8}$. La mayoría de los estudiantes sin dirección corresponde a casos en que la base de datos del SIGE tenía el campo de la dirección vacío, mientras que

${ }^{5}$ De acuerdo con datos del simce de 2010 relativos a cuarto grado, el $95,2 \%$ de los hogares devolvieron el cuestionario a la escuela. Para probar si hay diferencias significativas entre los estudiantes cuyos padres enviaron la información y los que no lo hicieron, se comparó el puntaje de lectura, matemática e historia del SIMCE de 2010 y el nivel socioeconómico de la escuela para ambos grupos. Los resultados indicaron que, en promedio, los estudiantes cuyos padres enviaron los cuestionarios obtuvieron 10 puntos más en lectura, 8,5 puntos más en matemática y 8,5 puntos más en historia que aquellos que no lo hicieron. También se encontró que los estudiantes cuyos hogares no devolvieron el cuestionario tendían a asistir a escuelas de nivel socioeconómico más bajo. Ambos resultados indican que los estudiantes de bajo nivel socioeconómico están ligeramente subrepresentados en la muestra.

${ }^{6} \mathrm{La}$ base de datos utilizada no contiene ningún otro dato personal que pueda revelar la identidad de un estudiante (por ejemplo, el nombre o el número de documento de identidad).

${ }^{7}$ La base de datos en formato .kmz (Google Earth) está disponible en línea en http://www.mineduc.cl.

${ }^{8}$ Las escuelas privadas no subvencionadas no se tuvieron en cuenta debido al bajo porcentaje de estudiantes con datos relativos a la dirección. La normalización, validación y geodecodificación (obtención de las coordenadas geográficas) de las direcciones fueron realizadas por Infomatic (http://www.infomatic.cl/). un porcentaje menor corresponde a direcciones que no pudieron localizarse ${ }^{9}$. Después de eliminar a los estudiantes que residían fuera del Gran Santiago, se obtuvo una muestra de 31.371 estudiantes distribuidos en 1.240 escuelas. Se utilizaron las coordenadas del lugar de residencia de cada estudiante y de todas las escuelas de Santiago para determinar la escuela más cercana. Dicho ejercicio se realizó para construir el escenario contrafactual. Por otra parte, se utilizó la información sobre la escuela a la que cada estudiante asistía para construir los índices de segregación real respecto de la misma muestra.

El nivel socioeconómico de cada estudiante (NSE) se determinó mediante el nivel de educación más alto obtenido por cualquiera de sus padres ${ }^{10}$. Sobre la base de esa información, los estudiantes se clasificaron en tres categorías: nivel socioeconómico bajo (menos de 12 años de educación o educación secundaria incompleta); nivel socioeconómico medio (12 años de educación o educación secundaria completa); y nivel socioeconómico alto (más de 12 años de educación o educación superior incompleta o completa).

Las escuelas se clasificaron según dos criterios. En primer lugar, se dividieron en tres categorías según el tipo de propiedad: i) públicas; ii) privadas subvencionadas con fines de lucro, y iii) privadas subvencionadas sin fines

\footnotetext{
${ }^{9}$ Una de las debilidades del sige es que no proporciona información precisa sobre el proceso de recolección de direcciones dentro de cada escuela, porque no es obligatorio recolectar esa información. Para determinar si había sesgos en la muestra de estudiantes con coordenadas, se realizaron pruebas de diferencias de media para los años de educación de los padres, el ingreso del hogar, los resultados del SIMCE y el tamaño del hogar entre los estudiantes con y sin coordenadas. Los resultados indican que las diferencias, aunque significativas en algunos casos, son de muy baja magnitud. Por otra parte, para ver los resultados por escuela, se calculó el promedio de años de educación de la madre por escuela, utilizando en primer lugar la muestra con coordenadas y luego la población total. En el $80 \%$ de las escuelas, la diferencia entre estas cifras es mayor que -1 (la muestra subestima el promedio de años de educación de la madre en menos de un año) y menor que 1 (la muestra sobrestima el promedio de años de educación de la madre en menos de un año).

${ }^{10}$ Es decir, los años de educación del padre o los años de educación de la madre. La educación de los padres se ha utilizado ampliamente en otras investigaciones para construir indicadores del nivel socioeconómico de los estudiantes. Véase una revisión de varias definiciones en Sirin (2005).
} 
de lucro ${ }^{11}$. En segundo lugar, las escuelas se dividieron en cuatro categorías según las barreras de entrada que establecían a las familias: i) bajo precio y baja selectividad; ii) alto precio y baja selectividad; iii) bajo precio y alta selectividad, y iv) alto precio y alta selectividad. Una escuela se considera de bajo precio cuando la escolaridad mensual cobrada durante el año escolar 2009 fue de 15 dólares o menos ${ }^{12}$. Una escuela se considera de baja

\footnotetext{
${ }^{11}$ Entre los propietarios con fines de lucro se incluyen personas naturales y jurídicas. En este último caso puede tratarse de sociedades anónimas, sociedades de responsabilidad limitada o empresas individuales de responsabilidad limitada. Entre los propietarios sin fines de lucro se incluyen organizaciones como fundaciones, congregaciones religiosas, asociaciones gremiales, sindicatos, asociaciones de vecinos, organizaciones comunitarias y cooperativas.

12 Las escuelas que cobran hasta 15 dólares son equivalentes a las escuelas gratuitas porque no se aplican descuentos al voucher por estudiante.
}

selectividad cuando está ubicada dentro de los primeros siete deciles de un índice construido con información sobre los requisitos y documentos que los padres deben presentar en el momento de matricular al estudiante, proveniente de la encuesta del SIMCE a los padres de los alumnos de cuarto grado ${ }^{13}$. En el cuadro 1 se presentan las estadísticas descriptivas de la muestra final.

CUADRO 1

Estadísticas descriptivas de la muestra final

(En porcentajes)

Nivel socioeconómico del estudiante

$\begin{array}{ll}\text { Bajo } & 25,1 \\ \text { Medio } & 39,6\end{array}$

Alto $\quad 35,3$

Tipo de propietario de la escuela

Pública

Privada subvencionada con fines de lucro $\quad 46,6$

$\begin{array}{ll}\text { Privada subvencionada sin fines de lucro } & 18,4\end{array}$

Barreras de entrada

Bajo precio y baja selectividad 52,8

Alto precio y baja selectividad 13,6

Bajo precio y alta selectividad 11,5

Alto precio y alta selectividad 22,1

Asiste a la escuela más cercana 19,1

Fuente: Elaboración propia.

\section{Resultados}

Los resultados se presentan en cuatro subsecciones. En la primera se comparan varios índices de segregación en el escenario real versus el escenario contrafactual. En las dos subsecciones siguientes se descompone el nivel de segregación entre tipos de escuela (diferencias en la proporción de estudiantes de distinto nivel socioeconómico que se matriculan en cada tipo de escuela) y dentro de cada tipo de escuela (diferencias en la distribución de los estudiantes dentro de cada sector). En la última subsección se examina la medida en que las barreras de entrada - pago de mensualidades y selección escolarinfluyen en los resultados. 


\section{Segregación real y segregación en el escenario contrafactual}

En el cuadro 2 se presenta una comparación entre diferentes índices de segregación socioeconómica en la situación real y en el escenario contrafactual. Resulta evidente que la segregación socioeconómica entre los estudiantes de nivel socioeconómico bajo es menor en el escenario contrafactual, dado que el índice de disimilitud entre esa categoría de estudiantes y el resto disminuye 12 puntos (de 0,481 a 0,360$)^{14}$. En términos de exposición, esto significa que el estudiante promedio de nivel socioeconómico bajo asiste a una escuela donde el $56 \%$ de sus compañeros tiene un nivel socioeconómico medio o alto, a pesar de que en todo el Gran Santiago el 75\% de los estudiantes pertenecen a uno de esos dos grupos (véase el cuadro 1). Cuando la distribución se basa exclusivamente en el lugar de residencia del estudiante, la exposición aumenta al 64,4\%.

Al examinar los resultados de los índices que comparan dos grupos (NSE bajo y NSE medio, NSE bajo

${ }^{14}$ Para analizar la sensibilidad de los índices a la muestra de estudiantes empleada, se comparó el índice de Duncan, Nivel socioeconómico bajo - Resto en la situación real $(0,481)$ con este mismo índice construido a partir de la población total (con y sin coordenadas). El valor del índice en este caso fue de 0,46. Estos resultados están disponibles a solicitud. y NSE alto, NSE medio y NSE alto), el mayor efecto se observa en la distribución de los dos grupos extremos (NSE alto y NSE bajo). En este caso, el índice de Duncan se reduce casi 15 puntos (de 0,657 a 0,511 ). Sin embargo, es interesante destacar que el índice de Duncan para los grupos de NSE bajo y NSE alto tiene un valor de 0,511 en el escenario contrafactual, lo que implica que el nivel de segregación residencial entre ambos grupos es elevado.

Por último, el índice de Duncan para múltiples grupos muestra un reducción de menor magnitud que el índice de Duncan NSE bajo - Resto (9 puntos), porque tiene en cuenta la distribución de los tres grupos simultáneamente. En este caso, el índice de Duncan disminuye de 0,417 a 0,332 . Esto sugiere que los estudiantes de los niveles socioeconómicos medio y alto pueden estar menos concentrados que los estudiantes de nivel socioeconómico bajo.

Estos resultados sugieren que la segregación es mayor en las escuelas que en los barrios. Tales hallazgos coinciden con los reportados por Bellei, De los Ríos y Valenzuela (2014), quienes usaron una metodología y datos diferentes. En las dos subsecciones siguientes se muestra que el menor nivel de segregación en el escenario contrafactual se explica por la reducción en el nivel de segregación entre los distintos tipos de escuela y en el interior de estos.

CUADRO 2

\section{Comparación de los índices de segregación en la situación real y en el escenario contrafactual}

Índice de segregación



Nivel socioeconómico bajo-Resto

Indice de disimilitud $(D)$

Índice de exposición $(X)$

Nivel socioeconómico bajo-Nivel socioeconómico medio

Índice de disimilitud $(D$

Índice de exposición $(X)$

Nivel socioeconómico bajo-Nivel socioeconómico alto

Indice de disimilitud $(D)$

Índice de exposición $(X)$

Nivel socioeconómico medio-Nivel socioeconómico alto

\begin{tabular}{lrr}
\hline Índice de disimilitud $(D)$ & 0,445 & 0,379 \\
Índice de exposición $(X)$ & 0,339 & 0,372
\end{tabular}

Múltiples grupos

Índice de Duncan (D)

0,417

0,332

Situación real

Fuente: Elaboración propia.

$\begin{array}{ll}0,362 & 0,274 \\ 0,501 & 0,544\end{array}$

Escenario contrafactual: todos los estudiantes asisten a la escuela más cercana a su lugar de residencia

0,481

$0,657 \quad 0,511$

$\begin{array}{ll}0,282 & 0,389\end{array}$ 


\section{Segregación entre sectores en la situación real y en el escenario contrafactual}

Consistente con la mayor parte de la literatura previa, en el gráfico 1 se muestra que en el sector público se matricula actualmente un porcentaje mayor de estudiantes de nivel socioeconómico bajo y un porcentaje menor de estudiantes de nivel socioeconómico alto que en el sector privado subvencionado. Sin embargo, dentro del sector privado, en el sector con fines de lucro se matricula un mayor porcentaje de estudiantes de nivel socioeconómico bajo y medio y un menor porcentaje de estudiantes de nivel socioeconómico alto que en el sector sin fines de lucro. Estos resultados coinciden con los presentados en Elacqua (2012).

En el escenario contrafactual, donde los estudiantes asisten a la escuela más cercana a su lugar de residencia, el porcentaje de estudiantes de nivel socioeconómico alto en el sector público aumentaría 10 puntos (de 19,9\% a $29,9 \%$ ), mientras que el porcentaje de estudiantes de nivel socioeconómico bajo disminuiría a su vez 10 puntos (de 40,0\% a 29,3\%). En contraste, el porcentaje de estudiantes de nivel socioeconómico bajo en el sector sin fines de lucro aumentaría 11 puntos (de $15,2 \%$ a $26,2 \%$ ) y el porcentaje de estudiantes de nivel socioeconómico alto disminuiría 16 puntos (de 50,2\% a $34,3 \%$ ). Los cambios son menos pronunciados en el sector con fines de lucro.

Estos resultados sugieren que en los sectores público y sin fines de lucro, las escuelas tienden sistemáticamente a matricular a un alumnado que no refleja la composición social del barrio en que están localizadas. En el caso de las escuelas públicas, estas matriculan estudiantes con un nivel socioeconómico inferior al del barrio, mientras que en el caso de las sin fines de lucro, estas matriculan estudiantes con un nivel socioeconómico superior al del barrio. El hecho de que la composición social de las escuelas en el sector con fines de lucro no cambie en forma significativa en ambos escenarios sugiere una mayor heterogeneidad en el interior de este sector.

GRÁFICO

Distribución de la matrícula en la situación real y en el escenario contrafactual, por tipo de escuela

(En porcentajes)

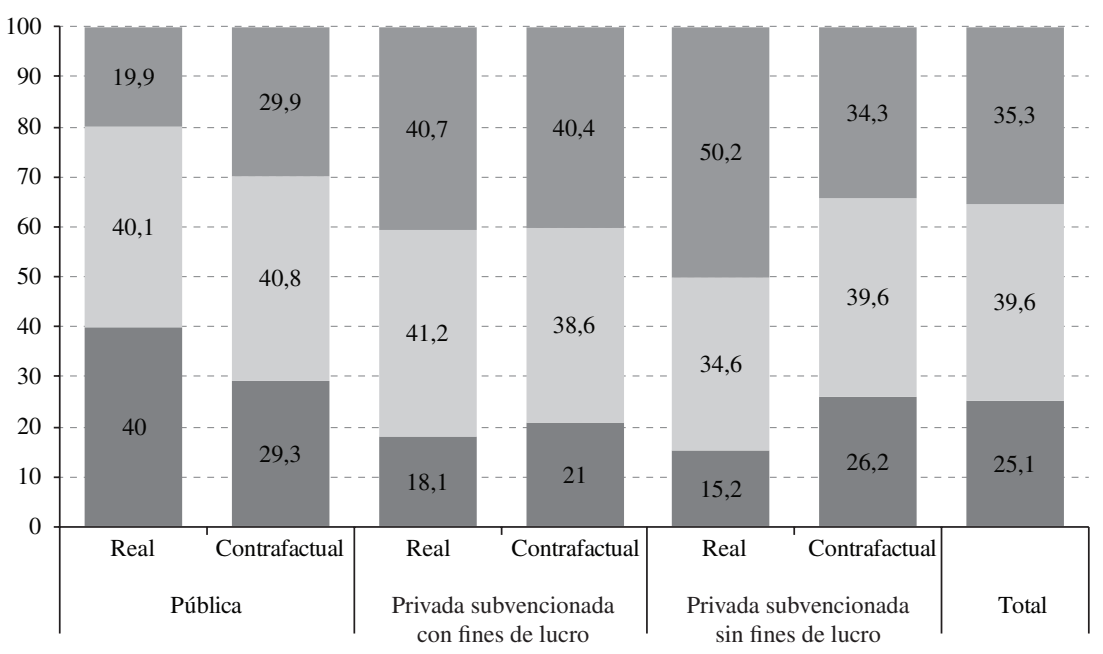

Nivel socioeconómico bajo

Nivel socioeconómico medio

Nivel socioeconómico alto

Fuente: Elaboración propia

\section{Segregación dentro de los sectores en la situación real y en el escenario contrafactual}

En el gráfico 2 se aprecia que la menor y la mayor segregación en términos de nivel socioeconómico se observan, respectivamente, en el sector público y en el sector sin fines de lucro.
Si bien la segregación socioeconómica disminuye en todos los sectores, en el escenario contrafactual el efecto es mayor entre las escuelas privadas subvencionadas, especialmente en el sector sin fines de lucro. La interpretación de estos cambios es interesante. En el escenario contrafactual, la distribución de los estudiantes de diferentes niveles socioeconómicos dentro de cada 
sector depende de la segregación residencial y la ubicación geográfica de las escuelas. En consecuencia, el nivel de segregación que persiste en este escenario se explica por el hecho de que las escuelas están situadas en barrios con diferente composición social. El mayor nivel de segregación en la situación real sugiere que las preferencias expresadas por los padres y las barreras de entrada establecidas por las escuelas aumentan la concentración de estudiantes de niveles socioeconómicos similares, más allá de lo que puede explicarse por la localización de las escuelas, especialmente en el sector sin fines de lucro.

GRÁFICO 2

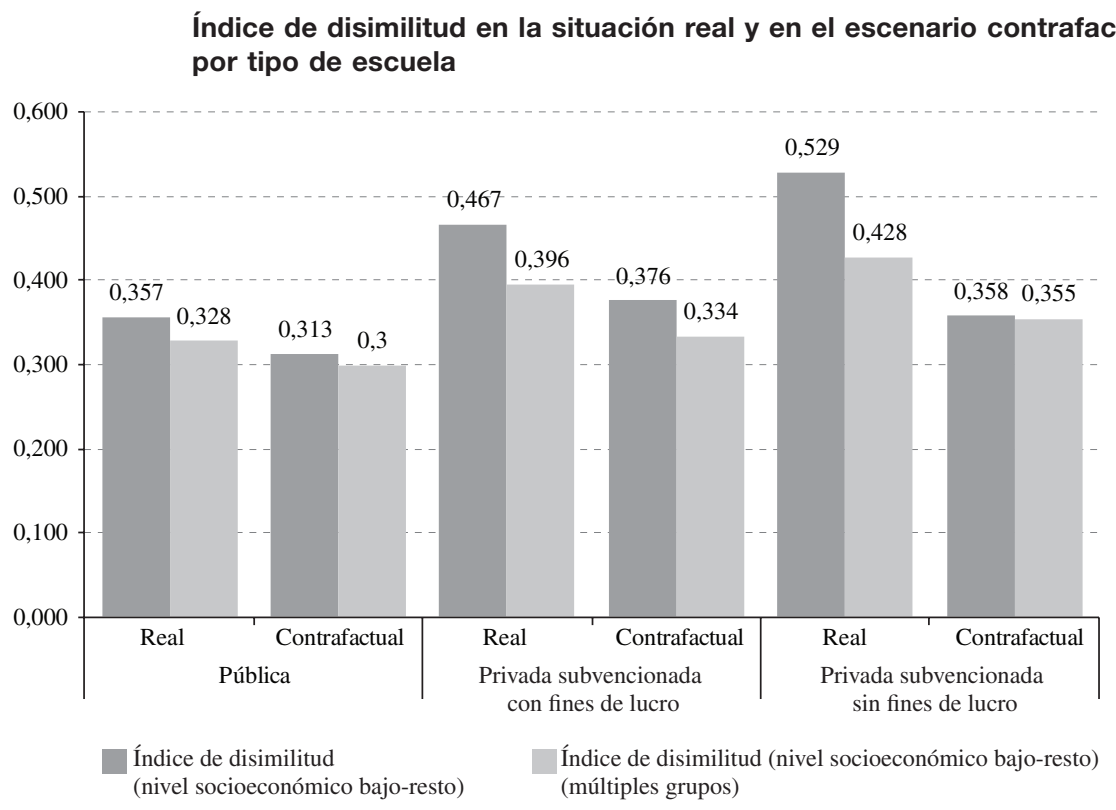

Fuente: Elaboración propia

\section{El papel de las barreras de entrada}

Para examinar la manera en que estos resultados se relacionan con las barreras de entrada establecidas por las escuelas, en esta subsección se categoriza a las escuelas de acuerdo con el monto de mensualidad que cobran y los requisitos que exigen a las familias durante el proceso de admisión. En el gráfico 3 se observa la distribución de las escuelas dentro de cada sector según las barreras de entrada. Se aprecia que el sector público tiene las barreras más bajas al ingreso, mientras que el sector subvencionado sin fines de lucro establece las más altas. Dentro del sector con fines de lucro se advierte más heterogeneidad.

Para analizar la forma en que la heterogeneidad dentro del sector con fines de lucro incide en la segregación escolar, en el gráfico 4 se compara la composición socioeconómica de las escuelas en la situación real $y$ en el escenario contrafactual. El eje $x$ corresponde al porcentaje de estudiantes de nivel socioeconómico bajo que se matricularía en la escuela en el escenario contrafactual, y el eje $y$ corresponde al porcentaje de estudiantes de esa categoría realmente matriculados. La línea de 45 grados representa el punto en que estas proporciones son iguales ${ }^{15}$. A fin de evitar valores extremos, el análisis se limita a las escuelas que tienen más de diez estudiantes en ambos casos ${ }^{16}$.

Por una parte, existe un grupo de escuelas (la mayoría de ellas en la categoría de baja selectividad y bajo precio) que, de manera análoga a las escuelas públicas, matriculan a un mayor porcentaje de estudiantes de nivel socioeconómico bajo que aquel que matricularían bajo el escenario contrafactual, lo que sugiere que compiten por los mismos estudiantes. Por otra parte, existe un conjunto de escuelas que matriculan a un porcentaje menor de estudiantes de nivel socioeconómico bajo que aquel en

15 Si todas las escuelas estuvieran sobre la línea de 45 grados la segregación real y la segregación hipotética serían iguales.

$16 \mathrm{Al}$ aplicar esta restricción queda el $62,4 \%$ de los estudiantes de la muestra. 
GRÁFICO 3

Tipos de escuela y barreras de entrada

(En porcentajes)

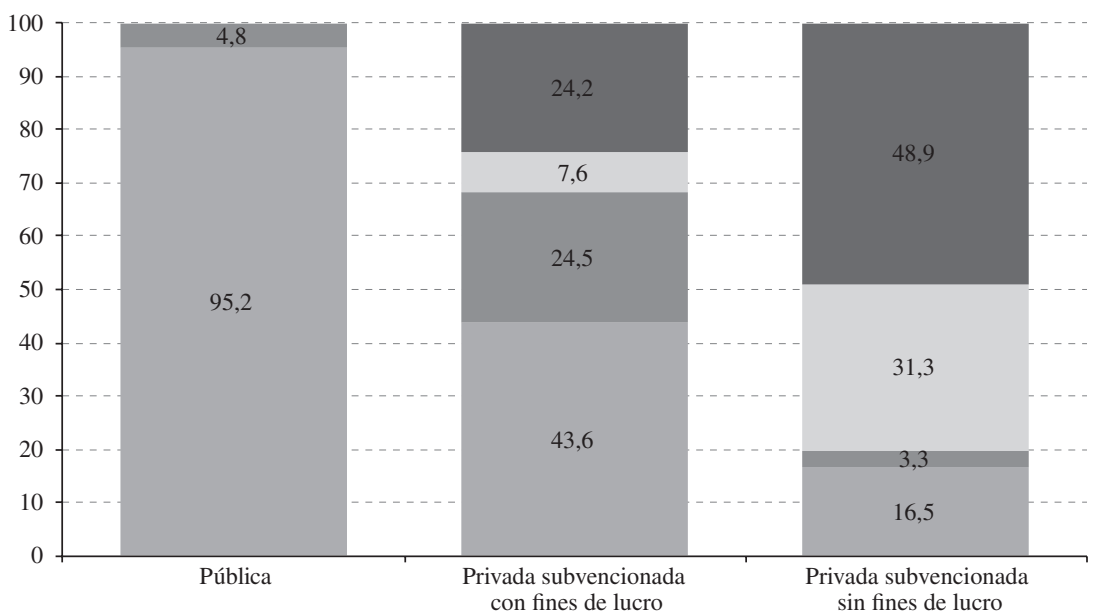

Bajo precio y baja selectividad

Alto precio y baja selectividad

Bajo precio y alta selectividad

Alto precio y alta selectividad

Fuente: Elaboración propia

GRÁFICO 4

Composición socioeconómica de las escuelas subvencionadas con fines de lucro en la situación real y en el escenario contrafactual, según las barreras de entrada

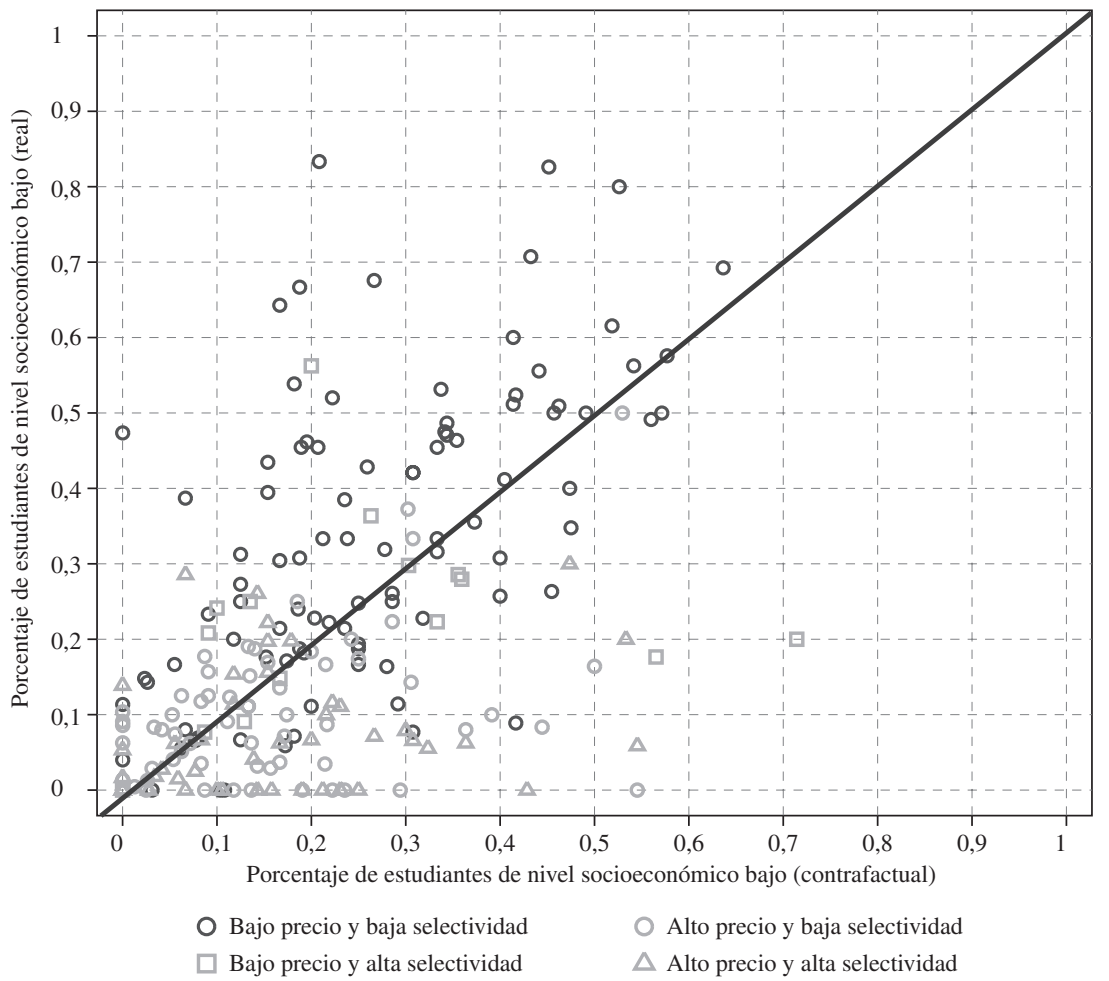

Fuente: Elaboración propia

Nota: El análisis se limita a las escuelas que tienen más de diez estudiantes en ambos casos. 
el escenario contrafactual. Este grupo está formado sobre todo por escuelas que cobran altas mensualidades y, en algunos casos, altamente selectivas (triángulos grises). Estos resultados coinciden con los de otros estudios en que se encuentra que el sector con fines de lucro tiende a buscar nichos de mercados entre familias de distintos niveles socioeconómicos por medio de la diferenciación de precios (Elacqua, 2012).

\section{VI}

\section{Conclusiones y repercusiones para la formulación de políticas públicas}

Con el propósito de aportar al debate sobre los efectos de la elección escolar en la segregación socioeconómica, en este artículo se utiliza una base de datos única y detallada de las direcciones de estudiantes y escuelas del Gran Santiago para comparar la segregación escolar real con la segregación que existiría en el caso hipotético de que los estudiantes asistieran a la escuela más cercana a su lugar de residencia.

En consonancia con estudios previos en otros países, los resultados indican que, independientemente del índice utilizado, la segregación escolar es mayor en la situación real que en el escenario contrafactual. Esto significa que la segregación es mayor en las escuelas que en los barrios y sugiere que la interacción entre las preferencias de las familias y las barreras de entrada establecidas por las escuelas incrementan la segregación escolar por sobre el efecto de la segregación residencial. Este hallazgo contradice el argumento de que la concentración de estudiantes de un nivel socioeconómico similar es simplemente un reflejo de la segregación residencial que existe en Santiago. Los resultados de este trabajo demuestran que hay escuelas cuya composición social es diferente de la del barrio en que están situadas.

Los menores niveles de segregación observados en el escenario contrafactual se pueden explicar por dos efectos complementarios. En primer lugar, si los estudiantes asistieran a la escuela más cercana a su lugar de residencia, la segregación entre los sectores público y sin fines de lucro disminuiría. Esto contradice el argumento de algunos propietarios de escuelas sin fines de lucro — sobre todo católicas — que sostienen que su menor proporción de estudiantes desfavorecidos deriva del creciente nivel socioeconómico de los barrios en que históricamente se han situado (Elacqua, 2012). En la práctica, el funcionamiento de la mayoría de estas escuelas es muy similar al que los críticos de la elección escolar atribuyen a las escuelas con fines de lucro, en la medida en que establecen barreras de entrada que excluyen a las familias de nivel socioeconómico bajo, incluso en barrios diversos desde el punto de vista socioeconómico. Este resultado no se limita al sistema educativo chileno. De acuerdo con varios estudios, la presión competitiva en los Estados Unidos de América, por ejemplo, induce a las escuelas sin fines de lucro (e incluso a algunas escuelas públicas) a utilizar estrategias de localización y admisión que excluyen a los estudiantes más desfavorecidos (véanse Lubienski, Gulosino y Weitzel, 2009; Miron, Urschel y Mathis, 2010).

En segundo lugar, los resultados indican que la segregación escolar dentro de los sectores con fines de lucro y sin fines de lucro disminuiría si la distribución de los estudiantes se basara exclusivamente en el lugar de residencia. Esto significa que las preferencias de los padres y las barreras de entrada aplicadas por las escuelas aumentan la concentración de estudiantes de nivel socioeconómico similar, más allá de lo que puede explicarse por la localización de las escuelas y la segregación residencial. La mayor caída en el sector sin fines de lucro se explica por el alto porcentaje de escuelas de este tipo que combinan precios elevados con procedimientos de admisión selectivos. Sin embargo, el sector con fines de lucro es casi tres veces mayor que el sector sin fines de lucro, de manera que los cambios en el primero tendrían efectos mayores en la segregación general.

El desafío en términos de la formulación de políticas para promover una distribución más equitativa de los estudiantes es que la segregación escolar deriva del equilibrio de decisiones individuales realizadas por varios agentes (familias y escuelas), que probablemente no internalizan los efectos agregados de sus acciones. Desde esta perspectiva, la segregación es una falla de mercado que justifica la acción del gobierno. Aunque no es uno de los objetivos de este artículo, determinar la medida 
en que este resultado es atribuible a las preferencias de los padres o a las decisiones estratégicas de las escuelas es clave para formular políticas públicas tendientes a reducir la segregación.

Desde el punto de vista de la oferta, el uso de barreras de entrada en las escuelas es claramente incompatible con la libertad de elección de los padres y los vouchers escolares. En otros países con políticas similares, como Bélgica, los Países Bajos y Suecia, la educación subvencionada (pública y privada) es gratuita y se prohíbe que las escuelas seleccionen a sus estudiantes. En consecuencia, para garantizar un sistema en que los padres tengan una verdadera libertad de elección, esas restricciones deberían eliminarse. Esta afirmación se basa en estudios recientes que muestran que la segregación escolar en Chile se explica en gran medida por la parte de la oferta (Flores y Carrasco, 2013; Arteaga, Paredes y Paredes, 2014). Por ejemplo, Flores y Carrasco (2013) sostienen que las preferencias expresadas por los padres dependen de las restricciones a la elección que enfrentan, sobre todo el precio de las escuelas locales, y que las diferencias en las preferencias por calidad entre padres de distintos niveles socioeconómicos disminuyen cuando se tienen en cuenta dichas restricciones (véase también Elacqua, Buckley y Schneider, 2006). Además, los costos de implementación de las políticas públicas orientadas a la oferta serían inferiores con respecto a los de las políticas que limitan las decisiones de las familias, pues la elección de escuelas se percibe como un derecho y ha sido una característica histórica del sistema educativo chileno (Aedo, 2000). Sin embargo, desde el punto de vista de la demanda, es importante mejorar la cantidad y la calidad de la información disponible para las familias, dado que la ausencia de esta es un factor que probablemente también fomenta la segregación escolar, más allá de las diferencias en las preferencias entre familias de distintos niveles socioeconómicos. En la mayoría de los sistemas basados en la elección escolar en todo el mundo se prevén sistemas de información para apoyar a las familias.

La pregunta fundamental es cómo eliminar de manera eficaz las barreras de entrada. Por una parte, los cobros obligatorios podrían eliminarse gradualmente. En comparación con otros países de la Organización para la Cooperación y el Desarrollo Económicos (OCDE), el gasto por estudiante en Chile es todavía bajo, a pesar del constante incremento registrado en los últimos 20 años. En consecuencia, el desafío consiste en reemplazar gradualmente los recursos privados aportados por las familias, preservando al mismo tiempo los recursos destinados a los estudiantes más desfavorecidos. Esta propuesta es coherente con una iniciativa legislativa recientemente promulgada por el Congreso Nacional (Cámara de Diputados de Chile, 2015) ${ }^{17}$. En cuanto al proceso de admisión, pese a que la Ley General de Educación (LGE) prohíbe la selección en la enseñanza primaria, la evidencia presentada en este artículo demuestra que el uso de mecanismos de selección continúa siendo una práctica común en las escuelas privadas subvencionadas. En algunas investigaciones recientes se sugiere que esto se debe a la falta de aplicación de la normativa (Carrasco y otros, 2014). En respuesta a este problema, el gobierno actual decidió crear un sistema centralizado para la admisión de los estudiantes en las escuelas subvencionadas (públicas y privadas). En este nuevo sistema, que se implementará gradualmente a partir de 2016, las familias deben elegir y clasificar las escuelas de su preferencia en una plataforma informática administrada por el MINEDUC. Cuando las escuelas elegidas por las familias tengan suficientes vacantes, todos los estudiantes serán admitidos automáticamente. En el caso de que las vacantes no sean suficientes para cubrir todas las solicitudes, las escuelas deberán utilizar un procedimiento de selección aleatorio (por ejemplo, un sorteo) para asegurar que no haya una selección arbitraria. En este proceso se dará prioridad a los estudiantes con hermanos ya matriculados en la escuela, a los niños desfavorecidos y a los hijos del personal de la escuela ${ }^{18}$. El sistema también tomará en cuenta las preferencias de los padres en el proceso de asignación de la escuela (Cámara de Diputados de Chile, 2015).

Aunque las características del sistema educativo chileno contribuyen a la segregación escolar, los resultados de esta investigación indican que la segregación residencial en el Gran Santiago también limita la integración de estudiantes de distintos niveles socioeconómicos. Por lo tanto, las políticas urbanas pueden desempeñar un papel fundamental en la reducción de la segregación escolar. Las políticas dirigidas a mejorar el acceso y la calidad del transporte público son igualmente importantes, pues esto influye en el número de opciones disponibles para las familias, en particular las más desfavorecidas (Asahi, 2014).

\footnotetext{
17 Naturalmente, al considerar el impacto social de esta política se debería tener en cuenta que los recursos públicos invertidos tienen un costo de oportunidad (por ejemplo, inversión en la educación preescolar). 18 Abdulkadiroğlu y Sönmez (2003) presentan la teoría económica y una revisión de varios distritos escolares de los Estados Unidos de América en los que se han adoptado procesos de admisión escolar centralizados.
} 


\section{Bibliografía}

Abdulkadiroğlu, A. y T. Sönmez (2003), "School choice: a mechanism design approach", American Economic Review, vol. 93, Nㅜ 3, Nashville, Tennessee, American Economic Association.

Aedo, R. (2000), La educación privada en Chile: un estudio históricoanalítico desde el período colonial hasta 1990, Santiago, RIL editores.

Allen, R. (2007), "Allocating pupils to their nearest secondary school: the consequences for social and ability stratification", Urban Studies, vol. 44, $\mathrm{N}^{\circ} 4$, SAGE.

Alves F. y otros (2012), "The effects of school choice on equity: evidence from Rio de Janeiro and Santiago", documento presentado en la reunión del ISA Research Committee on Social Stratification and Mobility (RC28) "Labor Market and Education Transitions in Uncertain Times", Charlottesville.

Arteaga, F., V. Paredes y R. Paredes (2014), "School segregation in Chile: residence, co-payment, or preferences?", inédito.

Asahi, K. (2014), "The impact of better school accessibility on student outcomes", SERC Discussion Paper, $\mathrm{N}^{\circ} 156$, Londres, Spatial Economics Research Centre, London School of Economics and Political Science [en línea] http://www.spatialeconomics. ac.uk/textonly/SERC/publications/download/sercdp0156.pdf.

Auguste, S., y J.P. Valenzuela (2004), "Do students benefit from school competition? Evidence from Chile", Ann Arbor, Universidad de Michigan, inédito.

Bayer, P., F. Ferreira y R. McMillan (2007), "A unified framework for measuring preferences for schools and neighborhoods", Journal of Political Economy, vol. 115, $\mathrm{N}^{\circ}$ 4, Chicago, University of Chicago Press.

Bellei, C. (2010), "Segregación socioeconómica y académica de la educación chilena: magnitud, causas y consecuencias", Santiago, Universidad de Chile, inédito.

Bellei, C., D. de los Ríos y J.P. Valenzuela (2014), "Socioeconomic school segregation in a market-oriented educational system: the case of Chile", Journal of Education Policy, vol. 29, $\mathrm{N}^{\circ} 2$, Taylor \& Francis.

Beyer, H. y S. Eyzaguirre (2014), "Fin del financiamiento compartido: análisis del proyecto de ley y propuestas", Puntos de Referencia, $\mathrm{N}^{\circ} 374$, Santiago, Centro de Estudios Públicos [en línea] http://www.cepchile.cl/Puntos-de-Referencia_CEP_PDF/ pder374_HBeyer-SEyzaguirre.pdf.

Bifulco, R., H. Ladd y S. Ross (2009), "Public school choice and integration evidence from Durham, North Carolina", Social Science Research, vol. 38, $\mathrm{N}^{\circ}$ 1, Amsterdam, Elsevier.

Black, S. (1999), "Do better schools matter? Parental valuation of elementary education", Quarterly Journal of Economics, vol. 114, $\mathrm{N}^{\circ} 2$, Oxford University Press.

Bustos, B., D. Contreras y P. Sepúlveda (2010), "When schools are the ones that choose: the effects of screening in Chile", Social Science Quarterly, vol. 91, $\mathrm{N}^{\circ}$ 5, Wiley.

Cámara de Diputados de Chile (2015), "Oficio N 131-362. Proyecto de Ley que regula la admisión de los y las estudiantes, elimina el financiamiento compartido y prohíbe el lucro en establecimientos educacionales que reciben aportes del Estado", Santiago.

Carrasco, A. y otros (2014), "Selección de estudiantes y desigualdad educacional en Chile: ¿Qué tan coactiva es la regulación que la prohíbe?", Proyecto FONIDE, $\mathrm{N}^{\circ} 711286$, Santiago, Ministerio de Educación.

Crain, R. y J. Strauss (1985), "School Desegregation and Black Occupational Attainments: Results from a Long-Term Experiment", Johns Hopkins University [en línea] http://eric. ed.gov/?id=ED260170

Duncan, O.D. y B. Duncan (1955), "A methodological analysis of segregation indexes", American Sociological Review, vol. 20, $\mathrm{N}^{\circ} 2$ [en línea] http://www.jstor.org/stable/2088328.
El Mercurio (2013), "Principales errores sobre financiamiento compartido", Santiago, 11 de julio [en línea] http://impresa. elmercurio.com.

Elacqua, G. (2012), "The impact of school choice and public policy on segregation: evidence from Chile", International Journal of Educational Development, vol. 32, No 3, Amsterdam, Elsevier.

Elacqua, G., J. Buckley y M. Schneider (2006), "School choice in Chile: is it class or classroom", Journal of Policy Analysis and Managment, vol. 25, $\mathrm{N}^{\circ} 3$, Wiley.

Elacqua, G. y R. Fábrega (2004), "El consumidor de la educación el actor olvidado de la libre elección de escuelas en Chile", Santiago, Programa de Promoción de la Reforma Educativa en América Latina y el Caribe (PREAL) [en línea] http://www.uai. cl/images/sitio/docentes/documentos/consumidor_educacion. pdf.

Elacqua, G., M. Martínez y H. Santos (2015), "Voucher policies and the response of for-profit and religious schools: evidence from Chile", Handbook of International Development and Education, P. Dixon, C. Counihan y S. Humble (eds.), Cheltenham, Edward Elgar.

(2011), "Lucro y educación escolar", Claves de Políticas Públicas, $\mathrm{N}^{\circ} 1$, Santiago, Instituto de Políticas Públicas, Universidad Diego Portales [en línea] http://www. politicaspublicas.udp.cl/publicaciones/detalle.tpl?id=285.

Fack, G. y J. Grenet (2010), "When do better schools raise housing prices? Evidence from Paris public and private schools", $C E E$ Discussion Paper, $\mathrm{N}^{\circ} 119$, Londres, Centre for the Economics of Education, London School of Economics [en línea] http:// cee.lse.ac.uk/ceedps/ceedp119.pdf

Flores, C. y A. Carrasco (2013), "(Des)igualdad de oportunidades para elegir escuela: preferencias, libertad de elección y segregación escolar", Documento de Referencia, $\mathrm{N}^{\circ}$ 2, Espacio Público [en línea] http://espaciopublico.cl/publicaciones/detalle.tpl?id=8.

Gallego, F. (2002), "Competencia y resultados educativos: teoría y evidencia para Chile", Cuadernos de Economía, vol. 39, $\mathrm{N}^{\circ} 118$ [en línea] http://repositorio.uc.cl/xmlui/ handle/123456789/4790.

Gallego, F. y A. Hernando (2009), "School choice in Chile: looking at the demand side", Documento de Trabajo, $\mathrm{N}^{\circ} 356$, Santiago, Instituto de Economía, Pontificia Universidad Católica [en línea] http://www.economia.puc.cl/DT?docid=3343.

(2008), "On the determinants and implications of school choice: semi-structural simulations for Chile", Documento de Trabajo, $\mathrm{N}^{\circ} 343$, Santiago, Instituto de Economía, Pontificia Universidad Católica [en línea] http://www.economia.puc.cl/ AR?docid=1197.

Gibbons, S. y S. Machin (2006), "Paying for primary schools: supply constraints, school popularity or congestion", The Economic Journal, vol. 116, $\mathrm{N}^{\circ} 510$, Wiley.

(2003), "Valuing English primary schools", Journal of Urban Economics, vol. 53, $\mathrm{N}^{\circ}$ 2, Amsterdam, Elsevier.

Guryan, J. (2004), "Desegregation and black dropout rates", American Economic Review, vol. 94, $\mathrm{N}^{\circ}$ 4, Nashville, Tennessee, American Economic Association [en línea] http://www.jstor. org/stable/3592799.

Hanushek, E., J. Kain y S. Rivkin (2009), "New evidence about Brown v. Board of Education: the complex effects of school racial composition on achievement", Journal of Labor Economics, vol. 27, $\mathrm{N}^{\circ} 3$, Chicago, University of Chicago Press.

(2004), "Why public schools lose teachers", Journal of Human Resources, vol. 39, $\mathrm{N}^{\circ}$ 2, Wisconsin, University of Wisconsin Press.

Hoxby, C. (2002), "The power of peers: how does the makeup of a classroom influence achievement", Education Next, vol. 2, $\mathrm{N}^{\circ} 2$ [en línea] http://educationnext.org/the-power-of-peers/. 
Hsieh, C. y M. Urquiola (2006), "The effects of generalized school choice on achievement and stratification: evidence from Chile's voucher program", Journal of Public Economics, vol. 90, $\mathrm{N}^{\circ}$ 8-9, Amsterdam, Elsevier.

James, E. (1993), "Why do different countries choose a different public-private mix of educational services?", The Journal of Human Resources, vol. 28, $\mathrm{N}^{\circ}$ 3, University of Wisconsin Press.

James, D. y K. Taeuber (1985), "Measures of segregation", Sociological Methodology, vol. 15, Wiley [en línea] http:// www.jstor.org/stable/270845.

Kahlenberg, R. (2001), All Together Now: Creating Middle Class Schools through Public School Choice, Washington, D.C., Brookings Institution Press.

Krysan, M. (2002), "Whites who say they'd flee: who are they, and why would they leave?", Demography, vol. $39, \mathrm{~N}^{\circ} 4$, Springer.

Levin, H.M. (2002), "Potential of for-profit schools for educational reform", Occasional Paper, $\mathrm{N}^{\circ}$ 47, Teachers College, Columbia University [en línea] http://ncspe.org/publications_files/179_ OP47.pdf.

Levinson, M. y S. Levinson (2003), "Getting religion: religion, diversity, and community in public and private schools", School Choice: The Moral Debate, A. Wolfe (ed.), Princeton, Princeton University Press.

Lubienski, C., C. Gulosino y P. Weitzel (2009), "School choice and competitive incentives: mapping the distribution of educational opportunities across local education markets", American Journal of Education, vol. 115, $\mathrm{N}^{\circ}$ 4, Chicago, University of Chicago Press.

Massey, D. y N. Denton (1988), "The dimensions of residential segregation", Social Forces, vol. 67, N 2, Oxford University Press.

Miron, G., J. Urschel y W. Mathis (2010), Schools without Diversity: Education Management Organizations, Charter Schools, and the Demographic Stratification of the American School System [en línea] http://nepc.colorado.edu/publication/schools-withoutdiversity.

Moe, T. (2001), "The politics of vouchers", Schools, Vouchers and the American Public, T. Moe (ed.), Washington, D.C., Brookings Institution Press.

oCDE (Organización para la Cooperación y el Desarrollo Económicos) (2011), Education at a Glance 2011: OECD Indicators, París, OECD Publishing.
Östh, J., E. Andersson y B. Malmberg (2013), "School choice and increasing performance difference: a counterfactual approach", Urban Studies, vol. 50, $\mathrm{N}^{\circ} 2$, SAGE.

Reardon, S. y G. Firebaugh (2002), "Measures of multigroup segregation", Sociological Methodology, vol. 32, $\mathrm{N}^{\circ}$ 1, Wiley.

Reback, R. (2005), "House prices and the provision of local public services: capitalization under school choice programs", Journal of Urban Economics, vol. 57, $\mathrm{N}^{\circ}$ 2, Amsterdam, Elsevier.

Riedel, A. y otros (2010), "School choice in German primary schools: how binding are school districts?", Journal for Educational Research Online, vol. 2, $\mathrm{N}^{\circ} 1$ [en línea] http://www.j-e-r-o. com/index.php/jero/article/viewFile/76/65.

Rose-Ackerman, S. (1996), "Altruism, nonprofits, and economic theory", Journal of Economic Literature, vol. 34, $\mathrm{N}^{\circ} 2$ [en línea] http://www.jstor.org/stable/2729219.

Rothstein, R. (2004), Class and Schools: Using Social, Economic, and Educational Reform to Close the Black-White Achievement Gap, Washington, D.C., Economic Policy Institute.

Sirin, S. (2005), "Socioeconomic status and academic achievement: a meta-analytic review of research", Review of Educational Research, vol. 75, $\mathrm{N}^{\circ} 3$, SAGE.

Sohoni, D. y S. Saporito (2009), "Mapping school segregation: using GIS to explore racial segregation between schools and their corresponding attendance areas", American Journal of Education, vol. 115, $\mathrm{N}^{\circ} 4$, Chicago, University of Chicago Press.

Wells, A.S. y otros (2008), Both Sides Now: the Story of School Desegregation's Graduates, Berkeley, University of California Press.

White, M.J. (1986), "Segregation and diversity measures in population distribution", Population Index, vol. 52, $\mathrm{N}^{\circ} 2$, Office of Population Research [en línea] http://www.jstor.org/ stable/3644339.

Zimmer, R.W. y E.F. Toma (2000), "Peer effects in private and public schools across countries", Journal of Policy Analysis and Management, vol. 19, $\mathrm{N}^{\circ} 1$, Wiley.

Zoloth, B.S. (1976), "Alternative measures of school segregation", Land Economics, vol. 52, $\mathrm{N}^{\circ} 3$ [en línea] http://www.jstor. org/stable/3145527. 